\title{
FINITE VOLUME SCHEME FOR MULTI-DIMENSIONAL DRIFT-DIFFUSION EQUATIONS AND CONVERGENCE ANALYSIS
}

\author{
Claire Chainais-Hillairet ${ }^{1}$, Jian-Guo Liu ${ }^{2}$ and Yue-Jun Peng ${ }^{1}$
}

\begin{abstract}
We introduce a finite volume scheme for multi-dimensional drift-diffusion equations. Such equations arise from the theory of semiconductors and are composed of two continuity equations coupled with a Poisson equation. In the case that the continuity equations are non degenerate, we prove the convergence of the scheme and then the existence of solutions to the problem. The key point of the proof relies on the construction of an approximate gradient of the electric potential which allows us to deal with coupled terms in the continuity equations. Finally, a numerical example is given to show the efficiency of the scheme.
\end{abstract}

Mathematics Subject Classification. 65M60, 76X05.

Received: September 27, 2002.

\section{INTRODUCTION}

In the mathematical modeling and numerical simulation of semiconductor devices, the drift-diffusion system is widely used [19]. This system consists of the continuity equations for particle densities and a Poisson equation for electrostatic potential. It can be derived from the Euler-Poisson equations when the relaxation time goes to 0 . The mathematical justification of this zero-relaxation-time limit has been rigorously performed in $[16,17]$. In the numerical context, it is much simpler to deal with the elliptic-parabolic coupled system of drift-diffusion equations than the Euler-Poisson hyperbolic system. Since these equations are of elliptic-parabolic type, it is natural to consider them in a bounded domain with initial and boundary conditions. The existence of solutions to these equations has been proved under natural assumptions. In some situation, the uniqueness of solutions is also obtained, see $[2,12,13,15]$.

Let $\Omega \subset \mathbb{R}^{d}(d \geq 1)$ be an open and bounded domain with boundary $\Gamma=\partial \Omega$. We suppose throughout this paper that $\Omega$ is a polygon. For general domain with smooth boundary, an approximation of the domain will be involved, see [9]. For $T>0$, we denote by $\Omega_{T}=(0, T) \times \Omega$. If we neglect the recombination-generation rate,

\footnotetext{
Keywords and phrases. Finite volume scheme, drift-diffusion equations, approximation of gradient.

${ }^{1}$ Laboratoire de Mathématiques Appliquées, CNRS UMR 6620, Université Blaise Pascal (Clermont-Ferrand 2),

63177 Aubière Cedex, France. e-mail: chainais@math.univ-bpclermont.fr, peng@math.univ-bpclermont.fr

2 Institute for Physical Science and Technology and Department of Mathematics, University of Maryland, College Park,

Maryland 20742, USA. e-mail: jliu@math.umd.edu
} 
the drift-diffusion system reads:

$$
\begin{gathered}
\partial_{t} N-\operatorname{div}\left(\nabla r_{N}(N)-N \nabla V\right)=0, \\
\partial_{t} P-\operatorname{div}\left(\nabla r_{P}(P)+P \nabla V\right)=0, \\
-\Delta V=P-N+C,
\end{gathered}
$$

in $\Omega_{T}$. Here $N$ and $P$ are the electron density and the positively charged holes density, $r_{N}$ and $r_{P}$ are the corresponding pressures and $V$ is the electrostatic potential. The function $C=C(x)$ is the prescribed doping profile characterizing the device under consideration. Equations (1.1)-(1.3) are supplemented by the following initial and Dirichlet boundary conditions:

$$
\begin{array}{cc}
t=0: N=N^{0}, \quad P=P^{0} & \text { in } \Omega, \\
N=\bar{N}, \quad P=\bar{P}, \quad V=\bar{V} \quad \text { on } \Sigma,
\end{array}
$$

where $\Sigma=(0, T) \times \Gamma, \bar{N}, \bar{P}$ and $\bar{V}$ are given functions defined in $\Omega_{T}$.

We want to point out that in general the mixed Dirichlet-Neumann problem with homogeneous Neumann boundary conditions are used. However, in our numerical scheme, it is easier to treat the Neumann boundary conditions than the Dirichlet boundary conditions. Indeed, the homogeneous Neumann boundary condition for $V$ implies that $d V_{\sigma}^{n}=0$ (see (2.13) for the definition of $d V_{\sigma}^{n}$ ). Thus some boundary terms disappear in the numerical scheme (2.10)-(2.12) and the convergence analysis of the scheme becomes simpler. For the sake of simplicity, we only use the Dirichlet boundary conditions in this paper.

There exists a wide literature on numerical schemes for the drift-diffusion equations. In the linear pressure case, a mixed exponential fitting finite element scheme has been successfully developed in $[4,5]$. The adaptation of this scheme to the nonlinear pressure case has been considered in $[1,14]$ and $[18]$ where numerical results are given in 1-D and 2-D respectively. The convergence of a finite volume scheme to (1.1)-(1.5) in one space dimension is given in [6].

The purpose of this paper is to prove the convergence of the finite volume scheme to (1.1)-(1.5) in several space dimensions. To this end, we suppose that the initial and boundary conditions are away from the vacuum sets where $N=0$ or $P=0$, and we show that this property is conserved for all time $t>0$. Thus the continuity equations (1.1) and (1.2) are non degenerate parabolic which allows us to perform the convergence analysis of the scheme for general pressure functions instead of $\gamma$-laws in one space dimension, see [6]. It turns out that there are serious difficulties to prove the convergence of the scheme in the case that the system (1.1)-(1.3) may be degenerate, since the continuity equations (1.1) and (1.2) change type from parabolic to hyperbolic at the degenerate points. Among them the main difficulty is to establish weak-BV estimates. In one space dimension, these estimates are obtained due to the regularity of the electric potential in $L^{\infty}\left(0, T ; W^{1, \infty}(\Omega)\right)$ which is no longer true in multi-dimensional case. Nevertheless, it is possible to prove the convergence of the finite volume scheme to the degenerate drift-diffusion equations subjected to the homogeneous Neumann boundary conditions. We refer to [11] for the weak-BV estimates to a nonlinear degenerate parabolic equations with coefficients satisfying a similar condition to the homogeneous Neumann boundary conditions. The convergence of the scheme for the degenerate drift-diffusion equations with less general pressure functions is analyzed in [8].

It should be pointed out that our analysis is close to that of [11]. However, there are at least two main differences between the analysis in [11] and our paper. First of all, the $L^{\infty}$ estimates are not so obvious as that of [11] since we deal with a system of equations instead of a scalar equation. Secondly, there are some coupled (convection) terms in the continuity equations (1.1)-(1.2) which are not involved in the problem in [11]. To treat them, we have to introduce an approximate gradient of the electric potential and prove its weak-* convergence in $L^{\infty}\left(0, T ; L^{2}(\Omega)\right)$. This step is crucial in the proof of convergence and it seems that this step cannot be avoided. Up to our knowledge, this is the first time that such an approximation is introduced in the finite volume scheme in several space dimensions. See [6] for that approximation in one-dimensional case. 
This paper is organized as follows. In the next section, we construct the approximate solution to (1.1)-(1.5) by the finite volume scheme and give the main result of the paper. Section 3 is devoted to the well-posedness of the approximate solution. We show the $L^{\infty}$ stability, the existence and uniqueness of the approximate solutions of the scheme. In Section 4, we establish the compactness of the approximate solutions based on the energy estimates in $L^{2}\left(0, T ; H^{1}(\Omega)\right)$ for the electric potential and the densities. The convergence of the scheme will be proved in Section 5. Finally, we give a numerical example in the last section. More numerical results can be found in $[6,7]$ and $[8]$ in one and two space dimensions respectively.

\section{NUMERICAL SCHEME AND MAIN RESUlT}

In order to define our finite volume scheme and prove its convergence to a weak solution of the problem (1.1)(1.5), we need the following hypotheses:

(H1) $N^{0}, P^{0} \in L^{\infty}(\Omega), \bar{N}, \bar{P} \in L^{\infty}\left(\Omega_{T}\right) \cap H^{1}\left(\Omega_{T}\right), \bar{V} \in L^{\infty}\left(0, T ; H^{1}(\Omega)\right)$;

(H2) there exist two constants $m>0$ and $M>0$ such that

$$
m \leq N^{0}, P^{0} \leq M \text { in } \Omega \text { and } m \leq \bar{N}, \bar{P} \leq M \text { in } \Omega_{T}
$$

(H3) $r_{N}, r_{P} \in C^{2}([0,+\infty))$ are strictly increasing on $(0,+\infty)$;

(H4) $C \in L^{\infty}(\Omega)$.

Conditions (H2) and (H3) imply that the system (1.1)-(1.3) is not degenerate at the initial time and on the boundary. This property will be conserved for all time, since $r_{N}^{\prime}$ and $r_{P}^{\prime}$ never vanish in the problem (see Lem. 3.1). Under assumptions (H1)-(H4), the function $(N, P, V)$ is called a solution of the problem (1.1)-(1.5) if it satisfies: $N, P \in L^{\infty}\left(\Omega_{T}\right)$,

$$
r_{N}(N)-r_{N}(\bar{N}), r_{P}(P)-r_{P}(\bar{P}), V-\bar{V} \in L^{\infty}\left(0, T ; H_{0}^{1}(\Omega)\right)
$$

and for all test functions $\phi \in C_{0}^{\infty}([0, T) \times \Omega)$ and $\psi \in \mathcal{D}\left(\Omega_{T}\right)$

$$
\begin{gathered}
\iint_{\Omega_{T}}\left(N \partial_{t} \phi-\nabla r_{N}(N) \cdot \nabla \phi+N \nabla V \cdot \nabla \phi\right) \mathrm{d} x \mathrm{~d} t+\int_{\Omega} N^{0}(x) \phi(0, x) \mathrm{d} x=0, \\
\iint_{\Omega_{T}}\left(P \partial_{t} \phi-\nabla r_{P}(P) \cdot \nabla \phi-P \nabla V \cdot \nabla \phi\right) \mathrm{d} x \mathrm{~d} t+\int_{\Omega} P^{0}(x) \phi(0, x) \mathrm{d} x=0, \\
\iint_{\Omega_{T}} \nabla V \cdot \nabla \psi \mathrm{d} x \mathrm{~d} t=\iint_{\Omega_{T}}(P-N+C) \psi \mathrm{d} x \mathrm{~d} t .
\end{gathered}
$$

Since the problem is not degenerate, it is easy to see that condition (2.1) is equivalent to

$$
N-\bar{N}, P-\bar{P}, V-\bar{V} \in L^{2}\left(0, T ; H_{0}^{1}(\Omega)\right) .
$$

Now we define the finite volume scheme to the problem (1.1)-(1.5). Let $\mathcal{T}$ be a regular and admissible mesh of the domain $\Omega$ (see [10]), constituting of open and convex polygons called control volumes with maximum size (diameter) $h$. For a control volume $K \in \mathcal{T}$, we denote by $\mathcal{N}_{K}$ the set of neighbours of $K$ and $\mathcal{E}_{\text {ext, } K}$ the set of edges of $K$ on the boundary $\Gamma$. We denote also $\sigma_{K L}=\bar{K} \cap \bar{L}$ for all $L \in \mathcal{N}_{K}$. The admissibility of $\mathcal{T}$ implies that $\bar{\Omega}=\cup_{K \in \mathcal{T}} \bar{K}, K \cap L=\emptyset$ if $K, L \in \mathcal{T}$ and $K \neq L$, and there exists a finite sequence of points $\left(x_{K}\right)_{K \in \mathcal{T}}$ such that $x_{K} \in K$ and the straight line $\overline{x_{K} x_{L}}$ is orthogonal to the edge $\sigma_{K L}$. Finally, we define

$$
\tau_{K, L}=\frac{m\left(\sigma_{K L}\right)}{d\left(x_{K}, x_{L}\right)} \quad \text { if } K, L \in \mathcal{T} \quad \text { and } \quad \tau_{\sigma}=\frac{m(\sigma)}{d\left(x_{K}, \Gamma\right)} \quad \text { if } \sigma \in \mathcal{E}_{\mathrm{ext}, K}
$$


where $m(\sigma)$ stands for the measure of $\sigma$ and $d(a, b)$ is the distance between $a$ and $b$. Let $k$ be the time step and $t^{n}=n k$. For $T>0$, we denote by $M_{T}=E(T / k)$ the integer part of $T / k$ and $\delta=\max (k, h)$.

The initial and boundary conditions are approximated as their $L^{2}$ projections. More precisely,

$$
\begin{gathered}
N_{K}^{0}=\frac{1}{m(K)} \int_{K} N^{0}(x) \mathrm{d} x, \quad P_{K}^{0}=\frac{1}{m(K)} \int_{K} P^{0}(x) \mathrm{d} x \\
N_{\sigma}^{n+1}=\frac{1}{k m(\sigma)} \int_{t^{n}}^{t^{n+1}} \int_{\sigma} \bar{N}(t, s) \mathrm{d} s \mathrm{~d} t, \quad P_{\sigma}^{n+1}=\frac{1}{k m(\sigma)} \int_{t^{n}}^{t^{n+1}} \int_{\sigma} \bar{P}(t, s) \mathrm{d} s \mathrm{~d} t \\
V_{\sigma}^{n}=\frac{1}{k m(\sigma)} \int_{t^{n}}^{t^{n+1}} \int_{\sigma} \bar{V}(t, s) \mathrm{d} s \mathrm{~d} t
\end{gathered}
$$

for all $K \in \mathcal{T}, \sigma \in \mathcal{E}_{\mathrm{ext}, K}$ and $0 \leq n \leq M_{T}$. Similarly, the doping profile is approximated by

$$
C_{K}=\frac{1}{m(K)} \int_{K} C(x) \mathrm{d} x, \quad K \in \mathcal{T} .
$$

The finite volume scheme for the continuity equations (1.1)-(1.2) and the Poisson equation is defined respectively by:

$$
\begin{aligned}
& m(K) \frac{N_{K}^{n+1}-N_{K}^{n}}{k}+\sum_{L \in \mathcal{N}_{K}} \tau_{K, L}\left[r_{N}\left(N_{K}^{n+1}\right)-r_{N}\left(N_{L}^{n+1}\right)\right] \\
& +\sum_{\sigma \in \mathcal{E}_{\text {ext }, K}} \tau_{\sigma}\left[r_{N}\left(N_{K}^{n+1}\right)-r_{N}\left(N_{\sigma}^{n+1}\right)\right]+\sum_{L \in \mathcal{N}_{K}}\left[\left(d V_{K, L}^{n}\right)^{+} N_{K}^{n+1}+\left(d V_{K, L}^{n}\right)^{-} N_{L}^{n+1}\right] \\
& +\sum_{\sigma \in \mathcal{E}_{\text {ext }, K}}\left[\left(d V_{\sigma}^{n}\right)^{+} N_{K}^{n+1}+\left(d V_{\sigma}^{n}\right)^{-} N_{\sigma}^{n+1}\right]=0, \\
& m(K) \frac{P_{K}^{n+1}-P_{K}^{n}}{k}+\sum_{L \in \mathcal{N}_{K}} \tau_{K, L}\left[r_{P}\left(P_{K}^{n+1}\right)-r_{P}\left(P_{L}^{n+1}\right)\right] \\
& +\sum_{\sigma \in \mathcal{E}_{\text {ext }, K}} \tau_{\sigma}\left[r_{P}\left(P_{K}^{n+1}\right)-r_{P}\left(P_{\sigma}^{n+1}\right)\right]+\sum_{L \in \mathcal{N}_{K}}\left[\left(-d V_{K, L}^{n}\right)^{+} P_{K}^{n+1}+\left(-d V_{K, L}^{n}\right)^{-} P_{L}^{n+1}\right] \\
& +\sum_{\sigma \in \mathcal{E}_{\text {ext }, K}}\left[\left(-d V_{\sigma}^{n}\right)^{+} P_{K}^{n+1}+\left(-d V_{\sigma}^{n}\right)^{-} P_{\sigma}^{n+1}\right]=0, \\
& -\sum_{L \in \mathcal{N}_{K}} d V_{K, L}^{n}-\sum_{\sigma \in \mathcal{E}_{\text {ext }, K}} d V_{\sigma}^{n}=m(K)\left(P_{K}^{n}-N_{K}^{n}+C_{K}\right),
\end{aligned}
$$

for all $K \in \mathcal{T}$ and $0 \leq n \leq M_{T}$, where $x^{+}=\max (x, 0), x^{-}=\min (x, 0)$ and

$$
d V_{K, L}^{n}=\tau_{K, L}\left(V_{L}^{n}-V_{K}^{n}\right) \text { and } d V_{\sigma}^{n}=\tau_{\sigma}\left(V_{\sigma}^{n}-V_{K}^{n}\right) \quad \text { if } \sigma \in \mathcal{E}_{\text {ext }, K} .
$$

Here the scheme (2.10)-(2.11) for $N$ and $P$ are Euler implicit in time which avoid the restriction of the time step of the form $k=O\left(h^{2}\right)$ in the explicit scheme. In (2.10)-(2.11), the convection term $\Delta V$ is treated semi implicit to avoid coupling between the dynamic equations and the kinetic equation to gain the efficiency in the computation. This treatment arises some new difficulty which was not discussed in [11]. In our Lemma 3.1, however, it is shown that this semi implicit treatment does not impose any stability constraint in the time step. Finally, the approximate solution $\left(N_{\delta}, P_{\delta}, V_{\delta}\right)$ to the problem (1.1)-(1.5) associated to the mesh $\mathcal{T}$ is defined as 
piecewise constant function by:

$$
N_{\delta}(t, x)=N_{K}^{n+1}, \quad P_{\delta}(t, x)=P_{K}^{n+1}, \quad V_{\delta}(t, x)=V_{K}^{n} \quad \forall(t, x) \in\left[t^{n}, t^{n+1}\right) \times K,
$$

where $\left\{\left(N_{K}^{n}, P_{K}^{n}, V_{K}^{n}\right), K \in \mathcal{T}, 0 \leq n \leq M_{T}\right\}$ is given by the scheme (2.5)-(2.13).

Now the definition of the scheme is finished. The purpose of this paper is to prove the following result.

Theorem 2.1. Let (H1)-(H4) hold and $\mathcal{T}$ be an admissible mesh of $\Omega$. Then there exists a unique approximate solution $\left(N_{\delta}, P_{\delta}, V_{\delta}\right)$ to the scheme (2.5)-(2.13), which converges (up to a subsequence) to $(N, P, V)$ as $\delta \rightarrow 0$, where $(N, P, V)$ is a solution to the problem (1.1)-(1.5) in the sense of (2.1)-(2.4).

\section{WELL-POSEDNESS OF THE SCHEME}

Since the finite volume scheme (2.5)-(2.13) is semi implicit in time, the existence and uniqueness of approximate solution should be shown. The aim of this section is to prove such a result. The proof of the existence is based on an $L^{\infty}$ estimate for $N_{\delta}$ and $P_{\delta}$. In the case $C=0$, this estimate has been obtained in one space dimension by means of the matrix analysis [6]. It is possible to apply the same technique to the multi dimensional case to prove the $L^{\infty}$ estimate. Here we give a direct proof of this result in the general case where $C \in L^{\infty}(\Omega)$. Note that we don't need the assumption (H2) in the following results. This means that the $L^{\infty}$ stability and the existence and uniqueness of solutions are also valid for the degenerate drift-diffusion equations.

We first show the $L^{\infty}$ stability of the scheme given by Lemma 3.1. It implies in particular that the continuity equations (1.1)-(1.2) are not degenerate for all time (see (3.6) below). The existence and uniqueness of solutions are stated in Theorem 3.1. Let

$$
\begin{gathered}
A=\max \left(\sup _{x \in \Omega} N^{0}(x), \sup _{x \in \Omega} P^{0}(x), \sup _{(t, s) \in \Sigma} \bar{N}(t, s), \sup _{(t, s) \in \Sigma} \bar{P}(t, s)\right), \\
a=\min \left(\inf _{x \in \Omega} N^{0}(x), \inf _{x \in \Omega} P^{0}(x), \inf _{(t, s) \in \Sigma} \bar{N}(t, s), \inf _{(t, s) \in \Sigma} \bar{P}(t, s)\right), \\
\bar{C}=\|C\|_{L^{\infty}(\Omega)}, \quad D_{T}=A \exp (\bar{C} T)+\bar{C} .
\end{gathered}
$$

Lemma 3.1. Assume (H1), (H3)-(H4) hold and $a \geq 0$. Then for all $K \in \mathcal{T}$ and all $n=0,1, \ldots, M_{T}$, we have

$$
a \exp (-\bar{C} T) \leq N_{K}^{n}, P_{K}^{n} \leq A \exp (\bar{C} T)
$$

provided that $k<D_{T}^{-1}$. In particular, if $C=0$, the maximum principle holds for $N_{\delta}$ and $P_{\delta}$, i.e. (3.4) becomes

$$
a \leq N_{K}^{n}, P_{K}^{n} \leq A .
$$

If in addition, (H2) holds, then $N_{\delta}$ and $P_{\delta}$ are strictly positive and (3.4) can be replaced by

$$
0<N_{1} \stackrel{\text { def }}{=} m \exp (-\bar{C} T) \leq N_{K}^{n}, P_{K}^{n} \leq M \exp (\bar{C} T) \stackrel{\text { def }}{=} N_{2} .
$$

Proof. It suffices to show (3.4). It will be carried out by induction on $n$. To this end, let us define for $n=0,1, \ldots, M_{T}$ :

and

$$
a_{n}=\min \left(\min _{K \in \mathcal{T}} N_{K}^{n}, \min _{\sigma \in \mathcal{E}_{\text {ext }}} N_{\sigma}^{n}\right), \quad A_{n}=\max \left(\max _{K \in \mathcal{T}} N_{K}^{n}, \max _{\sigma \in \mathcal{E}_{\text {ext }}} N_{\sigma}^{n}\right)
$$

$$
b_{n}=\min \left(\min _{K \in \mathcal{T}} P_{K}^{n}, \min _{\sigma \in \mathcal{E}_{\text {ext }}} P_{\sigma}^{n}\right), \quad B_{n}=\max \left(\max _{K \in \mathcal{T}} P_{K}^{n}, \max _{\sigma \in \mathcal{E}_{\text {ext }}} P_{\sigma}^{n}\right),
$$


where $\mathcal{E}_{\text {ext }}=\cup_{K \in \mathcal{T}} \mathcal{E}_{\text {ext }, K}$. Then (3.4) is a consequence of the following inequalities:

$$
\begin{array}{ll}
a(1+k \bar{C})^{-n} \leq a_{n}, & A_{n} \leq A(1-k \bar{C})^{-n}, \\
a(1+k \bar{C})^{-n} \leq b_{n}, & B_{n} \leq A(1-k \bar{C})^{-n},
\end{array}
$$

since for all $K \in \mathcal{T}$ and $n=0,1, \ldots, M_{T}, a_{n} \leq N_{K}^{n} \leq A_{n}, b_{n} \leq P_{K}^{n} \leq B_{n}$ and

$$
\exp (-\bar{C} T) \leq(1+k \bar{C})^{-n}, \quad(1-k \bar{C})^{-n} \leq \exp (\bar{C} T) .
$$

The inequalities (3.7) and (3.8) are obvious for $n=0$ due to the definition of $a, A, a_{n}, b_{n}, A_{n}$ and $B_{n}$. Suppose (3.7) and (3.8) hold for some $n>0$ and we want to show them for $n+1$.

We only show (3.7) for $n+1$ since the proof of (3.8) for $n+1$ is similar. Using the scheme (2.12) and the equality

$$
\left(d V_{K, L}^{n}\right)^{+} N_{K}^{n+1}+\left(d V_{K, L}^{n}\right)^{-} N_{L}^{n+1}=d V_{K, L}^{n} N_{K}^{n+1}+\left(-d V_{K, L}^{n}\right)^{+}\left(N_{K}^{n+1}-N_{L}^{n+1}\right),
$$

the scheme (2.10) can be rewritten as:

$$
\begin{aligned}
N_{K}^{n+1}= & N_{K}^{n}+\frac{k}{m(K)} \sum_{L \in \mathcal{N}_{K}}\left(\tau_{K, L}\left[r_{N}\left(N_{L}^{n+1}\right)-r_{N}\left(N_{K}^{n+1}\right)\right]+\left(-d V_{K, L}^{n}\right)^{+}\left[N_{L}^{n+1}-N_{K}^{n+1}\right]\right) \\
& +\frac{k}{m(K)} \sum_{\sigma \in \mathcal{E}_{\text {ext }, K}}\left(\tau_{\sigma}\left[r_{N}\left(N_{\sigma}^{n+1}\right)-r_{N}\left(N_{K}^{n+1}\right)\right]+\left(-d V_{\sigma}^{n}\right)^{+}\left[N_{\sigma}^{n+1}-N_{K}^{n+1}\right]\right) \\
& +k\left(P_{K}^{n}-N_{K}^{n}+C_{K}\right) N_{K}^{n+1} .
\end{aligned}
$$

We have to distinguish the following cases:

(i) If both $a_{n+1}$ and $A_{n+1}$ are reached on the boundary $\Gamma$, from the definition of $a, A$ and $A_{n}$, we have

$$
a(1+k \bar{C})^{-(n+1)} \leq a \leq a_{n+1}, \quad A_{n+1} \leq A \leq A(1-k \bar{C})^{-(n+1)},
$$

which is (3.7) for $n+1$.

(ii) If both $a_{n+1}$ and $A_{n+1}$ are reached in $\mathcal{T}$, there exist $K_{0}, K_{1} \in \mathcal{T}$ such that

$$
N_{K_{0}}^{n+1}=A_{n+1} \quad \text { and } \quad N_{K_{1}}^{n+1}=a_{n+1} .
$$

Since $r_{N}$ and $r_{P}$ are increasing, from (3.9), we obtain

$$
\begin{aligned}
& N_{K_{1}}^{n+1} \geq N_{K_{1}}^{n}+k N_{K_{1}}^{n+1}\left(P_{K_{1}}^{n}-N_{K_{1}}^{n}+C_{K_{1}}\right), \\
& N_{K_{0}}^{n+1} \leq N_{K_{0}}^{n}+k N_{K_{0}}^{n+1}\left(P_{K_{0}}^{n}-N_{K_{0}}^{n}+C_{K_{0}}\right),
\end{aligned}
$$

or equivalently

$$
\begin{aligned}
& {\left[1-k\left(P_{K_{1}}^{n}+C_{K_{1}}\right)\right] N_{K_{1}}^{n+1} \geq N_{K_{1}}^{n}\left(1-k N_{K_{1}}^{n+1}\right),} \\
& {\left[1-k\left(P_{K_{0}}^{n}+C_{K_{0}}\right)\right] N_{K_{0}}^{n+1} \leq N_{K_{0}}^{n}\left(1-k N_{K_{0}}^{n+1}\right) .}
\end{aligned}
$$

From $k<D_{T}^{-1}$ and the assumption of induction, we have for all $K \in \mathcal{T}$,

$$
1-k\left(P_{K}^{n}+C_{K}\right) \geq 1-k\left(B_{n}+\bar{C}\right) \geq 1-k D_{T}>0 .
$$

Therefore, (3.11) implies that

$$
1-k N_{K_{1}}^{n+1} \geq 1-k N_{K_{0}}^{n+1}>0 .
$$


Since

$$
\left[1-k\left(P_{K_{1}}^{n}+C_{K_{1}}\right)\right] N_{K_{1}}^{n+1} \leq\left(1-k a_{n}+k \bar{C}\right) N_{K_{1}}^{n+1}
$$

and

we obtain from (3.10) that

$$
\left(1-k N_{K_{1}}^{n+1}\right) N_{K_{1}}^{n} \geq a_{n}\left(1-k N_{K_{1}}^{n+1}\right)
$$

Hence, by the assumption of induction,

$$
a_{n} \leq(1+k \bar{C}) N_{K_{1}}^{n+1}
$$

$$
a_{n+1}=N_{K_{1}}^{n+1} \geq a(1+k \bar{C})^{-(n+1)} .
$$

Similarly, we have

$$
\left[1-k\left(P_{K_{0}}^{n}+C_{K_{0}}\right)\right] N_{K_{0}}^{n+1} \geq\left[1-k\left(A_{n}+\bar{C}\right)\right] N_{K_{0}}^{n+1}
$$

and

which gives, together with (3.11)

$$
N_{K_{0}}^{n}\left(1-k N_{K_{0}}^{n+1}\right) \leq A_{n}\left(1-k N_{K_{0}}^{n+1}\right)
$$

Hence, from (3.7)

$$
A_{n} \geq(1-k \bar{C}) N_{K_{0}}^{n+1}
$$

This shows (3.7) for $n+1$.

$$
A_{n+1}=N_{K_{0}}^{n+1} \leq A(1-k \bar{C})^{-(n+1)}
$$

(iii) If $a_{n+1}$ is reached on the boundary $\Gamma$ and $A_{n+1}$ is reached in $\mathcal{T}$, or $a_{n+1}$ is reached in $\mathcal{T}$ and $A_{n+1}$ is reached on the boundary $\Gamma$, (3.7) follows from a combination of the results in cases (i) and (ii). We omit the detail of the proof here since the techniques used are similar to that of (i) and (ii).

Theorem 3.1. Assume (H1), (H3)-(H4) hold, $a \geq 0$ and $k<D_{T}^{-1}$. Then the sequence $\left(N_{\delta}, P_{\delta}\right)_{\delta>0}$ is bounded in $L^{\infty}\left(\Omega_{T}\right)$, and there exists a unique solution $\left\{\left(N_{K}^{n}, P_{K}^{n}, V_{K}^{n}\right), K \in \mathcal{T}, 0 \leq n \leq M_{T}\right\}$ to the scheme (2.5)-(2.13).

Proof. The existence of solutions is done by induction on $n$. First of all, for $n=0,\left(N_{K}^{0}, P_{K}^{0}\right)_{K \in \mathcal{T}}$ is defined by (2.6). Then we determine $\left(V_{K}^{0}\right)_{K \in \mathcal{T}}$ by the equation (2.12) with the boundary conditions $(2.8)$ for $n=0$. It is clear that this solution $\left(V_{K}^{0}\right)_{K \in \mathcal{T}}$ of the linear Poisson equation is unique. Suppose now that $\left(N_{K}^{n}, P_{K}^{n}\right)_{K \in \mathcal{T}}$ is known for some $n>0$. Then we obtain as above a unique solution $\left(V_{K}^{n}\right)_{K \in \mathcal{T}}$ from (2.12) and (2.8). Therefore, $d V_{K, L}^{n}$ and $d V_{\sigma}^{n}$ are defined for all $K, L \in \mathcal{T}$ and $\sigma \in \mathcal{E}_{\text {ext, } K}$. The existence of $\left(N_{K}^{n+1}, P_{K}^{n+1}\right)_{K \in \mathcal{T}}$ to the equations (2.10)-(2.11) with the boundary conditions (2.7) is a consequence of the $L^{\infty}$ stability. It has been shown in [11] by the topological degree technique.

Now we turn to prove the uniqueness of solutions. By the analysis above, it suffices to show the uniqueness of solutions to the equations (2.10)-(2.11). Still by induction on $n$, we suppose that $\left(N_{K}^{n}, P_{K}^{n}\right)_{K \in \mathcal{T}}=\left(\tilde{N}_{K}^{n}, \tilde{P}_{K}^{n}\right)_{K \in \mathcal{T}}$ and $\left(N_{\sigma}^{n+1}, P_{\sigma}^{n+1}\right)_{\sigma \in \mathcal{E}_{\text {ext }, K}}=\left(\tilde{N}_{\sigma}^{n+1}, \tilde{P}_{\sigma}^{n+1}\right)_{\sigma \in \mathcal{E}_{\text {ext }, K}}$. Let $\left(N_{K}^{n+1}, P_{K}^{n+1}\right)_{K \in \mathcal{T}}$ and $\left(\tilde{N}_{K}^{n+1}, \tilde{P}_{K}^{n+1}\right)_{K \in \mathcal{T}}$ be two solutions of (2.10)-(2.11) with $\left(V_{K}^{n}\right)_{K \in \mathcal{T}}$ given by (2.12) and (2.8). Multiplying by $k \operatorname{sgn}\left(N_{K}^{n+1}-\tilde{N}_{K}^{n+1}\right)$ the equation obtained by subtraction of the scheme (2.10) for $N_{K}^{n+1}$ and $\tilde{N}_{K}^{n+1}$ and noting the monotonicity of $r_{N}$, we obtain:

$$
\begin{aligned}
m(K)\left|N_{K}^{n+1}-\tilde{N}_{K}^{n+1}\right| & +k \sum_{L \in \mathcal{N}_{\mathcal{K}}} \tau_{K, L}\left(\left|r_{N}\left(N_{K}^{n+1}\right)-r_{N}\left(\tilde{N}_{K}^{n+1}\right)\right|-\left|r_{N}\left(N_{L}^{n+1}\right)-r_{N}\left(\tilde{N}_{L}^{n+1}\right)\right|\right) \\
& +k \sum_{L \in \mathcal{N}_{\mathcal{K}}}\left[\left(d V_{K, L}^{n}\right)^{+}\left|N_{K}^{n+1}-\tilde{N}_{K}^{n+1}\right|+\left(d V_{K, L}^{n}\right)^{-}\left|N_{L}^{n+1}-\tilde{N}_{L}^{n+1}\right|\right] \\
& +k \sum_{\sigma \in \mathcal{E}_{\mathrm{ext}, K}}\left[\tau_{\sigma}\left|r_{N}\left(N_{K}^{n+1}\right)-r_{N}\left(\tilde{N}_{K}^{n+1}\right)\right|+\left(d V_{\sigma}^{n}\right)^{+}\left|N_{K}^{n+1}-\tilde{N}_{K}^{n+1}\right|\right] \leq 0 .
\end{aligned}
$$


Let us define

$$
\alpha_{K, L}=\left(d V_{K, L}^{n}\right)^{+}\left|N_{K}^{n+1}-\tilde{N}_{K}^{n+1}\right|+\left(d V_{K, L}^{n}\right)^{-}\left|N_{L}^{n+1}-\tilde{N}_{L}^{n+1}\right| .
$$

Since $d V_{L, K}^{n}=-d V_{K, L}^{n}$, we have $\left(d V_{L, K}^{n}\right)^{-}=-\left(d V_{K, L}^{n}\right)^{+}$, which yields $\alpha_{K, L}=-\alpha_{L, K}$. Therefore

$$
\sum_{K \in \mathcal{T}} \sum_{L \in \mathcal{N}_{\mathcal{K}}} \alpha_{K, L}=0
$$

Similarly,

$$
\sum_{K \in \mathcal{T}} \sum_{L \in \mathcal{N}_{\mathcal{K}}} \tau_{K, L}\left(\left|r_{N}\left(N_{K}^{n+1}\right)-r_{N}\left(\tilde{N}_{K}^{n+1}\right)\right|-\left|r_{N}\left(N_{L}^{n+1}\right)-r_{N}\left(\tilde{N}_{L}^{n+1}\right)\right|\right)=0 .
$$

The last term on the left hand side of (3.12) is positive. Hence,

$$
\sum_{K \in \mathcal{T}} \sum_{L \in \mathcal{N}_{\mathcal{K}}} m(K)\left|N_{K}^{n+1}-\tilde{N}_{K}^{n+1}\right| \leq 0
$$

and thus $N_{K}^{n+1}=\tilde{N}_{K}^{n+1}$ for all $K \in \mathcal{T}$. In the same way, we obtain $P_{K}^{n+1}=\tilde{P}_{K}^{n+1}$ for all $K \in \mathcal{T}$. This shows the uniqueness of solutions to (2.10)-(2.11).

\section{Compactness of the approximate solution}

This section is devoted to the compactness of the approximate solution. Our goal is to show the strong compactness of the sequences $\left(N_{\delta}\right)_{\delta>0}$ and $\left(P_{\delta}\right)_{\delta>0}$ in $L^{2}\left(\Omega_{T}\right)$ and the weak-* compactness in $L^{\infty}\left(0, T ; L^{2}(\Omega)\right)$ of the approximate gradient of the electric potential which will be defined below. The first one is easy. It is based on a discrete $L^{2}\left(0, T ; H^{1}(\Omega)\right)$ estimate and an estimate of time translation. The second is the main task of this section.

From now on, we denote by $D_{j}(j=1,2, \ldots)$ various constants depending possibly on the given data and independent of $\delta, n$ and $K$. We first show the discrete $L^{\infty}\left(0, T ; H^{1}(\Omega)\right)$ estimate for $V_{\delta}$ and the $L^{2}\left(0, T ; H^{1}(\Omega)\right)$ estimates for $N_{\delta}$ and $P_{\delta}$. They are given by (4.1) and (4.2)-(4.3) respectively.

Lemma 4.1. Assume (H1)-(H4) hold and $k<D_{T}^{-1}$. Then there exist two constants $D_{1}>0$ and $D_{2}>0$ such that for all $n=1,2, \ldots, M_{T}$,

$$
\left\|V_{\delta}\right\|_{1, n, \Omega}^{2}=\sum_{K \in \mathcal{T}}\left(\frac{1}{2} \sum_{L \in \mathcal{N}_{\mathcal{K}}} \tau_{K, L}\left(V_{K}^{n}-V_{L}^{n}\right)^{2}+\sum_{\sigma \in \mathcal{E}_{\mathrm{ext}, K}} \tau_{\sigma}\left(V_{K}^{n}-V_{\sigma}^{n}\right)^{2}\right) \leq D_{1},
$$

and

$$
\left\|N_{\delta}\right\|_{1, \Omega_{T}}^{2}=\sum_{n=0}^{M_{T}} k \sum_{K \in \mathcal{T}}\left(\frac{1}{2} \sum_{L \in \mathcal{N}_{\mathcal{K}}} \tau_{K, L}\left(N_{K}^{n+1}-N_{L}^{n+1}\right)^{2}+\sum_{\sigma \in \mathcal{E}_{\text {ext }, K}} \tau_{\sigma}\left(N_{K}^{n+1}-N_{\sigma}^{n+1}\right)^{2}\right) \leq D_{2},
$$

and similarly

$$
\left\|P_{\delta}\right\|_{1, \Omega_{T}}^{2}=\sum_{n=0}^{M_{T}} k \sum_{K \in \mathcal{T}}\left(\frac{1}{2} \sum_{L \in \mathcal{N}_{\mathcal{K}}} \tau_{K, L}\left(P_{K}^{n+1}-P_{L}^{n+1}\right)^{2}+\sum_{\sigma \in \mathcal{E}_{\mathrm{ext}, K}} \tau_{\sigma}\left(P_{K}^{n+1}-P_{\sigma}^{n+1}\right)^{2}\right) \leq D_{2} .
$$


Proof. We first show (4.1). Let us define the discrete function $\bar{V}_{\delta}$ of $\bar{V}$ in $\Omega_{T}$ by $\bar{V}_{\delta}(t, x)=\bar{V}_{K}^{n}, \forall(t, x) \in$ $\left[t^{n}, t^{n+1}\right) \times K$, where

$$
\bar{V}_{K}^{n}=\frac{1}{k m(K)} \int_{t^{n}}^{t^{n+1}} \int_{K} \bar{V}(t, x) \mathrm{d} x \mathrm{~d} t, \quad \forall K \in \mathcal{T}, \quad n=0,1, \ldots, M_{T}
$$

Let $f_{K}^{n}=m(K)\left(P_{K}^{n}-N_{K}^{n}+C_{K}\right)$. Multiplying the scheme (2.12) by $V_{K}^{n}-\bar{V}_{K}^{n}$ and summing over $\mathcal{T}$, we obtain

$$
-\sum_{K \in \mathcal{T}}\left(\sum_{L \in \mathcal{N}_{K}} d V_{K, L}^{n}+\sum_{\sigma \in \mathcal{E}_{\text {ext }, K}} d V_{\sigma}^{n}\right)\left(V_{K}^{n}-\bar{V}_{K}^{n}\right)=\sum_{K \in \mathcal{T}} f_{K}^{n}\left(V_{K}^{n}-\bar{V}_{K}^{n}\right) .
$$

Noting that $\bar{V}_{\sigma}^{n}=V_{\sigma}^{n}$, it is easy to see from the $L^{\infty}$ estimates for $N_{\delta}$ and $P_{\delta}$ and Young inequality that

$$
\begin{aligned}
&-\sum_{K \in \mathcal{T}} \sum_{L \in \mathcal{N}_{K}} d V_{K, L}^{n}\left(V_{K}^{n}-\bar{V}_{K}^{n}\right)- \sum_{K \in \mathcal{T}} \sum_{\sigma \in \mathcal{E}_{\text {ext }, K}} d V_{\sigma}^{n}\left(V_{K}^{n}-\bar{V}_{K}^{n}\right)= \\
& \frac{1}{2} \sum_{K \in \mathcal{T}} \sum_{L \in \mathcal{N}_{K}} \tau_{K, L}\left(V_{K}^{n}-V_{L}^{n}\right)\left[\left(V_{K}^{n}-V_{L}^{n}\right)-\left(\bar{V}_{K}^{n}-\bar{V}_{L}^{n}\right)\right] \\
& \quad+\sum_{K \in \mathcal{T}} \sum_{\sigma \in \mathcal{E}_{\text {ext }, K}} \tau_{\sigma}\left(V_{K}^{n}-V_{\sigma}^{n}\right)\left[\left(V_{K}^{n}-V_{\sigma}^{n}\right)-\left(\bar{V}_{K}^{n}-\bar{V}_{\sigma}^{n}\right)\right] \\
& \geq \frac{1}{2}\left\|V_{\delta}\right\|_{1, n, \Omega}^{2}-\frac{1}{2}\left\|\bar{V}_{\delta}\right\|_{1, n, \Omega}^{2}
\end{aligned}
$$

and

$$
\left|f_{K}^{n}\left(V_{K}^{n}-\bar{V}_{K}^{n}\right)\right| \leq\left(2 N_{2}+\bar{C}\right)\left[\frac{1}{4 \varepsilon} m(K)+\varepsilon m(K)\left(V_{K}^{n}-\bar{V}_{K}^{n}\right)^{2}\right],
$$

where $\varepsilon>0$ is a real number, $\bar{C}$ and $N_{2}$ are defined by (3.3) and (3.6) respectively. Therefore

$$
\frac{1}{2}\left\|V_{\delta}\right\|_{1, n, \Omega}^{2} \leq \frac{1}{2}\left\|\bar{V}_{\delta}\right\|_{1, n, \Omega}^{2}+\frac{\left(2 N_{2}+\bar{C}\right) m(\Omega)}{4 \varepsilon}+\varepsilon\left(2 N_{2}+\bar{C}\right) \sum_{K \in \mathcal{T}} m(K)\left(V_{K}^{n}-\bar{V}_{K}^{n}\right)^{2}
$$

By choosing $\varepsilon$ small enough, we obtain (4.1) from $\bar{V} \in L^{\infty}\left(0, T ; H^{1}(\Omega)\right)$ and the discrete Poincaré inequality (see Lem. 3.1 of [10]).

The proof of (4.2) and (4.3) is almost the same. Hence, we only show (4.2). For simplicity, the subscript $N$ in $r_{N}$ will be omitted in the proof. As above, let us define the discrete function $\bar{N}_{\delta}$ of $\bar{N}$ in $\Omega_{T}$ by $\bar{N}_{\delta}(t, x)=\bar{N}_{K}^{n+1}, \forall(t, x) \in\left[t^{n}, t^{n+1}\right) \times K$, where

$$
\bar{N}_{K}^{n+1}=\frac{1}{k m(K)} \int_{t^{n}}^{t^{n+1}} \int_{K} \bar{N}(t, x) \mathrm{d} x \mathrm{~d} t, \quad \forall K \in \mathcal{T}, \quad n=0,1, \ldots, M_{T}
$$

Multiplying the scheme (2.10) by $k\left(N_{K}^{n+1}-\bar{N}_{K}^{n+1}\right)$ and summing for $K \in \mathcal{T}$ and $n=0,1, \ldots, M_{T}$, we obtain

$$
E_{1}(\delta)+E_{2}(\delta)+E_{3}(\delta)=0
$$


with

$$
\begin{aligned}
E_{1}(\delta)= & \sum_{n=0}^{M_{T}} \sum_{K \in \mathcal{T}} m(K)\left(N_{K}^{n+1}-N_{K}^{n}\right)\left(N_{K}^{n+1}-\bar{N}_{K}^{n+1}\right), \\
E_{2}(\delta)= & \sum_{n=0}^{M_{T}} k \sum_{K \in \mathcal{T}} \sum_{L \in \mathcal{N}_{\mathcal{K}}} \tau_{K, L}\left(r\left(N_{K}^{n+1}\right)-r\left(N_{L}^{n+1}\right)\right)\left(N_{K}^{n+1}-\bar{N}_{K}^{n+1}\right) \\
& +\sum_{n=0}^{M_{T}} k \sum_{K \in \mathcal{T}} \sum_{\sigma \in \mathcal{E}_{\mathrm{ext}, K}} \tau_{\sigma}\left(r\left(N_{K}^{n+1}\right)-r\left(N_{\sigma}^{n+1}\right)\right)\left(N_{K}^{n+1}-\bar{N}_{K}^{n+1}\right), \\
E_{3}(\delta)= & \sum_{n=0}^{M_{T}} k \sum_{K \in \mathcal{T}} \sum_{L \in \mathcal{N}_{\mathcal{K}}}\left[\left(d V_{K, L}^{n}\right)^{+} N_{K}^{n+1}+\left(d V_{K, L}^{n}\right)^{-} N_{L}^{n+1}\right]\left(N_{K}^{n+1}-\bar{N}_{K}^{n+1}\right) \\
& +\sum_{n=0}^{M_{T}} k \sum_{K \in \mathcal{T}} \sum_{\sigma \in \mathcal{E}_{\mathrm{ext}, K}}\left[\left(d V_{\sigma}^{n}\right)^{+} N_{K}^{n+1}+\left(d V_{\sigma}^{n}\right)^{-} N_{\sigma}^{n+1}\right]\left(N_{K}^{n+1}-\bar{N}_{K}^{n+1}\right) .
\end{aligned}
$$

For the term $E_{1}(\delta)$, we write $E_{1}(\delta)=E_{11}(\delta)+E_{12}(\delta)$ where,

$$
\begin{aligned}
& E_{11}(\delta)=\sum_{n=0}^{M_{T}} \sum_{K \in \mathcal{T}} m(K)\left(N_{K}^{n+1}-N_{K}^{n}\right) N_{K}^{n+1}, \\
& E_{12}(\delta)=\sum_{n=0}^{M_{T}} \sum_{K \in \mathcal{T}} m(K)\left(N_{K}^{n+1}-N_{K}^{n}\right) \bar{N}_{K}^{n+1} .
\end{aligned}
$$

From the inequality $t(t-s) \geq \frac{1}{2}\left(t^{2}-s^{2}\right), t, s \in \mathbb{R}$, we have

$$
\begin{aligned}
E_{11}(\delta) & \geq \frac{1}{2} \sum_{n=0}^{M_{T}} \sum_{K \in \mathcal{T}} m(K)\left[\left(N_{K}^{n+1}\right)^{2}-\left(N_{K}^{n}\right)^{2}\right] \\
& \geq-\frac{1}{2} \sum_{K \in \mathcal{T}} m(K)\left(N_{K}^{0}\right)^{2} \\
& \geq-\frac{1}{2} m(\Omega)\left\|N^{0}\right\|_{L^{\infty}(\Omega)}^{2}
\end{aligned}
$$

and a straightforward computation gives

$$
\begin{aligned}
E_{12}(\delta) & =\sum_{n=0}^{M_{T}} \sum_{K \in \mathcal{T}} m(K) N_{K}^{n}\left(\bar{N}_{K}^{n+1}-\bar{N}_{K}^{n}\right)+\sum_{K \in \mathcal{T}} m(K)\left(N_{K}^{M_{T}+1} \bar{N}_{K}^{M_{T}+1}-N_{K}^{0} \bar{N}_{K}^{1}\right) \\
& \geq-\left\|N_{\delta}\right\|_{L^{\infty}\left(\Omega_{T}\right)} \sum_{n=0}^{M_{T}} \sum_{K \in \mathcal{T}} m(K)\left|\bar{N}_{K}^{n+1}-\bar{N}_{K}^{n}\right|-m(\Omega)\left\|N_{\delta}\right\|_{L^{\infty}\left(\Omega_{T}\right)}\left\|\bar{N}_{\delta}\right\|_{L^{\infty}\left(\Omega_{T}\right)} .
\end{aligned}
$$

From the assumption $\bar{N} \in L^{\infty}\left(\Omega_{T}\right) \cap H^{1}\left(\Omega_{T}\right)$, we obtain $E_{1}(\delta) \geq-D_{3}$. For the term $E_{2}(\delta)$, we denote by $r_{m}=\min _{N \in\left[N_{1}, N_{2}\right]} r^{\prime}(N)$, where $N_{1}$ and $N_{2}$ are defined by (3.6). Since $r$ is strictly increasing on $(0,+\infty)$ and $N_{1}>0$, we have $r_{m}>0$. Hence, the estimate for $E_{2}(\delta)$ can be obtained in the similar way as (4.4). It is:

$$
E_{2}(\delta) \geq \frac{1}{2} r_{m}\left\|N_{\delta}\right\|_{1, \Omega_{T}}^{2}-D_{4} .
$$


Now we control the coupled term $E_{3}$. For this purpose, noting that $d V_{K, L}^{n}=-d V_{L, K}^{n}$ and $\bar{N}_{\sigma}^{n+1}=N_{\sigma}^{n+1}$, we obtain

$$
\begin{aligned}
E_{3}(\delta)= & \frac{1}{2} \sum_{n=0}^{M_{T}} k \sum_{K \in \mathcal{T}} \sum_{L \in \mathcal{N}_{\mathcal{K}}}\left[\left(d V_{K, L}^{n}\right)^{+} N_{K}^{n+1}+\left(d V_{K, L}^{n}\right)^{-} N_{L}^{n+1}\right]\left[\left(N_{K}^{n+1}-N_{L}^{n+1}\right)-\left(\bar{N}_{K}^{n+1}-\bar{N}_{L}^{n+1}\right)\right] \\
& +\sum_{n=0}^{M_{T}} k \sum_{K \in \mathcal{T}} \sum_{\sigma \in \mathcal{E}_{\text {ext }, K}}\left[\left(d V_{\sigma}^{n}\right)^{+} N_{K}^{n+1}+\left(d V_{\sigma}^{n}\right)^{-} N_{\sigma}^{n+1}\right]\left[\left(N_{K}^{n+1}-N_{\sigma}^{n+1}\right)-\left(\bar{N}_{K}^{n+1}-\bar{N}_{\sigma}^{n+1}\right)\right] .
\end{aligned}
$$

From the definition of $d V_{K, L}^{n}$, we have

$$
\begin{aligned}
\left|E_{3}(\delta)\right| \leq & \frac{1}{2}\left\|N_{\delta}\right\|_{L^{\infty}\left(\Omega_{T}\right)} \sum_{n=0}^{M_{T}} k \sum_{K \in \mathcal{T}} \sum_{L \in \mathcal{N}_{\mathcal{K}}} \tau_{K, L}\left|V_{L}^{n}-V_{K}^{n}\right|\left(\left|N_{K}^{n+1}-N_{L}^{n+1}\right|+\left|\bar{N}_{K}^{n+1}-\bar{N}_{L}^{n+1}\right|\right) \\
& +\left\|N_{\delta}\right\|_{L^{\infty}\left(\Omega_{T}\right)} \sum_{n=0}^{M_{T}} k \sum_{K \in \mathcal{T}} \sum_{\sigma \in \mathcal{E}_{\text {ext }, K}} \tau_{\sigma}\left|V_{\sigma}^{n}-V_{K}^{n}\right|\left(\left|N_{K}^{n+1}-N_{\sigma}^{n+1}\right|+\left|\bar{N}_{K}^{n+1}-\bar{N}_{\sigma}^{n+1}\right|\right) .
\end{aligned}
$$

It follows from Young and Cauchy-Schwarz inequality and (4.1) that

$$
E_{3}(\delta) \geq-\left\|N_{\delta}\right\|_{L^{\infty}\left(\Omega_{T}\right)}\left(\varepsilon\left\|N_{\delta}\right\|_{1, \Omega_{T}}^{2}+\frac{1}{4 \varepsilon} T D_{1}\right)-\left\|N_{\delta}\right\|_{L^{\infty}\left(\Omega_{T}\right)} \sqrt{T D_{1}}\left\|\bar{N}_{\delta}\right\|_{1, \Omega_{T}},
$$

where $\varepsilon>0$ is an arbitrary real number. Let us choose $\varepsilon$ such that $2 \varepsilon\left\|N_{\delta}\right\|_{L^{\infty}\left(\Omega_{T}\right)}<r_{m}$. Then the first term on the right hand side of (4.7) can be controlled by $\frac{1}{2} r_{m}\left\|N_{\delta}\right\|_{1, \Omega_{T}}^{2}$ in (4.6). Thus (4.2) follows from (4.5)-(4.7) and $E_{1}(\delta) \geq-D_{3}$.

Lemma 4.2. Let $\xi \in(0, T)$ and $\eta \in \Omega$. Under the assumptions of Lemma 4.1, there is a constant $D_{5}>0$ independent of $\delta$, $\xi$ and $\eta$ such that

$$
\begin{aligned}
\left\|N_{\delta}(.+\xi, .+\eta)-N_{\delta}(., .)\right\|_{L^{2}((0, T-\xi) \times \omega)}^{2} \leq D_{5}[\xi+|\eta|(|\eta|+h+1)], \\
\left\|P_{\delta}(.+\xi, .+\eta)-P_{\delta}(., .)\right\|_{L^{2}((0, T-\xi) \times \omega)}^{2} \leq D_{5}[\xi+|\eta|(|\eta|+h+1)],
\end{aligned}
$$

where $\omega$ is an open subdomain of $\Omega$ satisfying $x+\eta \in \Omega$ for all $x \in \omega$.

Lemma 4.2 is a consequence of Lemma 4.1 and the estimates of time translation for $N_{\delta}$ and $P_{\delta}$ obtained from the scheme (2.10)-(2.12). Its proof will be omitted here since it is similar to that of Lemma 4.3 and Lemma 4.6 in [10]. We refer to that book for the detail of the proof. See also [6] for the one-dimensional case. By Riesz-Fréchet-Kolmogorov criterion of strong compactness [3] together with (4.8)-(4.9), we obtain

Lemma 4.3. There exists a subsequence of $\left(N_{\delta}, P_{\delta}\right)_{\delta>0}$, still denoted by $\left(N_{\delta}, P_{\delta}\right)_{\delta>0}$, and a function $(N, P) \in$ $L^{\infty}\left(\Omega_{T}\right)$ such that as $\delta \rightarrow 0$,

$$
N_{\delta} \longrightarrow N \text { and } P_{\delta} \longrightarrow P \text { in } L^{2}\left(\Omega_{T}\right) \text { strongly } .
$$

Now we study the compactness of the sequence $\left(V_{\delta}\right)_{\delta>0}$. To this end, we have to define an approximation of the gradient of the electric potential $V$. Following the notations in Section 2, let $K \in \mathcal{T}$ and $L \in \mathcal{N}_{K}$ with common vertexes $\left(y_{K, L}^{j}\right)_{1 \leq j \leq J}\left(J \in \mathbb{N}^{*}\right)$. Let $T_{K, L}\left(T_{K, \sigma}\right.$ respectively, $\left.\sigma \in \mathcal{E}_{\text {ext }, K}\right)$ be the open and convex polygon with vertexes $\left(x_{K}, x_{L}\right)\left(x_{K}\right.$ respectively) and $\left(y_{K, L}^{j}\right)_{1 \leq j \leq J}$. Then the domain $\Omega$ can be decomposed as:

$$
\bar{\Omega}=\cup_{K \in \mathcal{T}}\left(\left(\cup_{L \in \mathcal{N}_{\mathcal{K}}} \bar{T}_{K, L}\right) \cup\left(\cup_{\sigma \in \mathcal{E}_{\text {ext }, K}} \bar{T}_{K, \sigma}\right)\right) .
$$


The approximation $d V_{\delta}$ of $\nabla V$ is defined as piecewise constants in $\Omega_{T}$ by:

$$
d V_{\delta}(t, x)= \begin{cases}\frac{m\left(\sigma_{K L}\right)\left(V_{L}^{n}-V_{K}^{n}\right)}{m\left(T_{K, L}\right)} \nu_{K, L} & \text { if }(t, x) \in\left(t^{n}, t^{n+1}\right) \times T_{K, L}, \\ \frac{m(\sigma)\left(V_{\sigma}^{n}-V_{K}^{n}\right)}{m\left(T_{K, \sigma}\right)} \nu_{K, \Gamma} & \text { if }(t, x) \in\left(t^{n}, t^{n+1}\right) \times T_{K, \sigma},\end{cases}
$$

for all $K \in \mathcal{T}$ and $0 \leq n \leq M_{T}$, where $\nu_{K, L}$ ( $\nu_{K, \Gamma}$ respectively) stands for the normal unit vector of $\sigma_{K L}\left(\nu_{K, \Gamma}\right.$ respectively) oriented from $K$ to $L$ (from $K$ to $\Gamma$ respectively). Similarly, we define $d r_{N}\left(N_{\delta}\right)$ and $d r_{P}\left(P_{\delta}\right)$ by

$$
\begin{aligned}
& d r_{N}\left(N_{\delta}\right)(t, x)= \begin{cases}\frac{m\left(\sigma_{K L}\right)\left(r_{N}\left(N_{L}^{n}\right)-r_{N}\left(N_{K}^{n}\right)\right)}{m\left(T_{K, L}\right)} \nu_{K, L} & \text { if }(t, x) \in\left(t^{n}, t^{n+1}\right) \times T_{K, L}, \\
\frac{m(\sigma)\left(r_{N}\left(N_{\sigma}^{n}\right)-r_{N}\left(N_{K}^{n}\right)\right)}{m\left(T_{K, \sigma}\right)} \nu_{K, \Gamma} & \text { if }(t, x) \in\left(t^{n}, t^{n+1}\right) \times T_{K, \sigma},\end{cases} \\
& d r_{P}\left(P_{\delta}\right)(t, x)= \begin{cases}\frac{m\left(\sigma_{K L}\right)\left(r_{P}\left(P_{L}^{n}\right)-r_{P}\left(P_{K}^{n}\right)\right)}{m\left(T_{K, L}\right)} \nu_{K, L} & \text { if }(t, x) \in\left(t^{n}, t^{n+1}\right) \times T_{K, L}, \\
\frac{m(\sigma)\left(r_{P}\left(P_{\sigma}^{n}\right)-r_{P}\left(P_{K}^{n}\right)\right)}{m\left(T_{K, \sigma}\right)} \nu_{K, \Gamma} & \text { if }(t, x) \in\left(t^{n}, t^{n+1}\right) \times T_{K, \sigma} .\end{cases}
\end{aligned}
$$

Then we have:

Lemma 4.4. There exists a function $V \in L^{\infty}\left(0, T ; H^{1}(\Omega)\right)$ such that, up to subsequences of $\left(V_{\delta}\right)_{\delta>0}$ and $\left(d V_{\delta}\right)_{\delta>0}$,

$$
V_{\delta} \longrightarrow V \quad \text { in } L^{\infty}\left(0, T ; L^{2}(\Omega)\right) \text { weakly -* as } \delta \rightarrow 0
$$

and

$$
d V_{\delta} \longrightarrow \nabla V \quad \text { in }\left(L^{\infty}\left(0, T ; L^{2}(\Omega)\right)\right)^{d} \text { weakly -* as } \delta \rightarrow 0 .
$$

Proof. Since the straight line $\overline{x_{K} x_{L}}$ is orthogonal to the edge $\sigma_{K L}$ for all $L \in \mathcal{N}_{K}$ and the mesh $\mathcal{T}$ is regular, there is a constant $D_{6}>0$ depending only on the dimension of the domain and the geometry of $\mathcal{T}$ such that

$$
d\left(x_{K}, x_{L}\right) m\left(\sigma_{K L}\right) \leq D_{6} m\left(T_{K, L}\right) .
$$

It follows from the definition of $d V_{\delta}$ and Lemma 4.1, the sequence $\left(d V_{\delta}\right)_{\delta>0}$ is bounded in $\left(L^{\infty}\left(0, T ; L^{2}(\Omega)\right)\right)^{d}$ and by the discrete Poincaré inequality, the sequence $\left(V_{\delta}\right)_{\delta>0}$ is bounded in $L^{\infty}\left(0, T ; L^{2}(\Omega)\right)$. Therefore, there exist two functions $V \in L^{\infty}\left(0, T ; L^{2}(\Omega)\right)$ and $\chi \in\left(L^{\infty}\left(0, T ; L^{2}(\Omega)\right)\right)^{d}$ such that (4.14) holds and

$$
d V_{\delta} \longrightarrow \chi \quad \text { in }\left(L^{\infty}\left(0, T ; L^{2}(\Omega)\right)\right)^{d} \text { weakly }-* \text { as } \delta \rightarrow 0 .
$$

It remains to show that $\chi=\nabla V$ in the sense of distributions, which is a consequence of the following limit:

$$
\forall \phi \in\left(\mathcal{D}\left(\Omega_{T}\right)\right)^{d}, \quad E_{\delta} \stackrel{\text { def }}{=} \iint_{\Omega_{T}} d V_{\delta} . \phi \mathrm{d} x \mathrm{~d} t+\iint_{\Omega_{T}} V_{\delta} \operatorname{div} \phi \mathrm{d} x \mathrm{~d} t \longrightarrow 0 \quad \text { as } \delta \rightarrow 0 .
$$


Let $\delta>0$ be small enough such that $\operatorname{supp} \phi \in(0, T) \times\{x \in \Omega ; d(x, \Gamma)>\delta\}$. This implies that $\phi$ vanishes in $T_{K, \sigma}$ for all $\sigma \in \mathcal{E}_{\text {ext }, K}$ and $K \in \mathcal{T}$. In view of $\nu_{K, L}=-\nu_{L, K}$, we obtain for all $t \in\left(t^{n}, t^{n+1}\right)$,

$$
\begin{aligned}
\int_{\Omega} V_{\delta}(t, x) \operatorname{div} \phi(t, x) \mathrm{d} x & =\sum_{K \in \mathcal{T}} \int_{K} V_{\delta}(t, x) \operatorname{div} \phi(t, x) \mathrm{d} x \\
& =\sum_{K \in \mathcal{T}} \sum_{L \in \mathcal{N}_{K}} V_{K}^{n} \int_{\sigma_{K L}} \phi(t, s) \cdot \nu_{K, L} \mathrm{~d} s \\
& =\frac{1}{2} \sum_{K \in \mathcal{T}} \sum_{L \in \mathcal{N}_{K}}\left(V_{K}^{n}-V_{L}^{n}\right) \int_{\sigma_{K L}} \phi(t, s) \cdot \nu_{K, L} \mathrm{~d} s
\end{aligned}
$$

and from the definition of $d V_{\delta}$,

$$
\begin{aligned}
\int_{\Omega} d V_{\delta}(t, x) \phi(t, x) \mathrm{d} x & =\frac{1}{2} \sum_{K \in \mathcal{T}} \sum_{L \in \mathcal{N}_{K}} \int_{T_{K, L}} d V_{\delta}(t, x) \cdot \phi(t, x) \mathrm{d} x \\
& =\frac{1}{2} \sum_{K \in \mathcal{T}} \sum_{L \in \mathcal{N}_{K}}\left(V_{L}^{n}-V_{K}^{n}\right) \frac{m\left(\sigma_{K L}\right)}{m\left(T_{K, L}\right)} \int_{T_{K, L}} \phi(t, x) \cdot \nu_{K, L} \mathrm{~d} x .
\end{aligned}
$$

Since $\phi$ is smooth, it is easy to see that for all $t \in(0, T)$,

$$
\left|\frac{1}{m\left(T_{K, L}\right)} \int_{T_{K, L}} \phi(t, x) \cdot \nu_{K, L} \mathrm{~d} x-\frac{1}{m\left(\sigma_{K L}\right)} \int_{\sigma_{K L}} \phi(t, s) \cdot \nu_{K, L} \mathrm{~d} s\right| \leq h\|\phi\|_{C^{1}\left(\overline{\Omega_{T}}\right)} .
$$

Therefore, by Cauchy-Schwarz inequality and (4.1)

$$
\begin{aligned}
2\left|E_{\delta}\right| & \leq h\|\phi\|_{C^{1}\left(\overline{\Omega_{T}}\right)} \sum_{n=0}^{M_{T}} k \sum_{K \in \mathcal{T}} \sum_{L \in \mathcal{N}_{K}}\left|V_{K}^{n}-V_{L}^{n}\right| m\left(\sigma_{K L}\right) \\
& \leq h \sqrt{T D_{1}}\|\phi\|_{C^{1}\left(\overline{\Omega_{T}}\right)}\left(\sum_{n=0}^{M_{T}} k \sum_{K \in \mathcal{T}} \sum_{L \in \mathcal{N}_{K}} d\left(x_{K}, x_{L}\right) m\left(\sigma_{K L}\right)\right)^{1 / 2} .
\end{aligned}
$$

Noting that

$$
\sum_{K \in \mathcal{T}} \sum_{L \in \mathcal{N}_{K}} m\left(T_{K, L}\right)=2 m(\Omega)
$$

we obtain from (4.16) and Lemma 4.1 that

$$
\left|E_{\delta}\right| \leq \frac{1}{2} h T\|\phi\|_{C^{1}\left(\overline{\Omega_{T}}\right)} \sqrt{2 D_{1} D_{6} m(\Omega)},
$$

and the result follows.

Corollary 4.1. There exist subsequences of $\left(d r_{N}\left(N_{\delta}\right)\right)_{\delta>0}$ and $\left(d r_{P}\left(P_{\delta}\right)\right)_{\delta>0}$ such that, as $\delta \rightarrow 0$,

$$
d r_{N}\left(N_{\delta}\right) \longrightarrow \nabla r_{N}(N), \quad d r_{P}\left(P_{\delta}\right) \longrightarrow \nabla r_{P}(P) \quad \text { in }\left(L^{2}\left(\Omega_{T}\right)\right)^{d} \text { weakly. }
$$

Proof. From the strong compactness of the sequence $\left(N_{\delta}\right)_{\delta>0}$ in $L^{2}\left(\Omega_{T}\right)$, we have, up to a subsequence of $\left(N_{\delta}\right)_{\delta>0}, \lim _{\delta \rightarrow 0} r_{N}\left(N_{\delta}\right)=r_{N}(N)$ in $L^{2}\left(\Omega_{T}\right)$. Since the sequence $\left(d r_{N}\left(N_{\delta}\right)\right)_{\delta>0}$ is bounded in $L^{2}\left(\Omega_{T}\right)$, the proof of Lemma 4.4 can be applied to the sequence $\left(d r_{N}\left(N_{\delta}\right)\right)_{\delta>0}$. The proof for the sequence $\left(d r_{P}\left(P_{\delta}\right)\right)_{\delta>0}$ is similar. 


\section{Convergence of the scheme}

In this section, we show the convergence of the approximate solutions $\left(N_{\delta}, P_{\delta}, V_{\delta}\right)$ to a global solution $(N, P, V)$ of the problem (1.1)-(1.5). The key point is to treat the nonlinear diffusion terms and the convection terms. The strong compactness of the sequence $\left(N_{\delta}, P_{\delta}\right)_{\delta>0}$ and the weak-* convergence of the sequence $\left(d V_{\delta}\right)_{\delta>0}$ allow to pass to the limit in the nonlinear diffusion terms and the convection terms. Therefore, the convergence of the scheme is the main task of this section. It will be achieved for the Poisson equation and the continuity equations in Theorem 5.1 and Theorem 5.2 separately. In particular, it implies the results of Theorem 2.1. Note that the convergence proof is simpler than those of [10] and [11] due to the introduction of the quantity $d V_{\delta}$ in Section 4. Moreover, we don't need the condition of type $k=O(\sqrt{h})$ used for the degenerate problem in one space dimension $[6]$.

Theorem 5.1. Assume (H1)- $\left(H_{4}\right)$ hold. Then the functions $(N, P, V)$ defined in Lemmas 4.3-4.4 satisfy the Poisson equation in the sense of (2.4) and the boundary condition $V-\bar{V} \in L^{\infty}\left(0, T ; H_{0}^{1}(\Omega)\right)$.

Proof. Let $\psi \in \mathcal{D}\left(\Omega_{T}\right)$ be a test function and $\psi_{K}^{n}=\psi\left(t^{n}, x_{K}\right)$ for all $K \in \mathcal{T}$ and $n=0,1, \ldots, M_{T}$. As in the proof of Lemma 4.4, we suppose that $\delta>0$ is small enough such that $\operatorname{supp} \psi \in(0, T) \times\{x \in \Omega ; d(x, \Gamma)>\delta\}$. This implies that $\psi$ vanishes in all $K \in \mathcal{T}$ when $\bar{K} \cap \Gamma \neq \emptyset$. It follows that $\sum_{\sigma \in \mathcal{E}_{\text {ext }, K}} g_{K}^{n} \psi_{K}^{n}=0$ for all $g_{K}^{n}$. We introduce:

where $C_{\delta}$ is defined by

$$
F_{10}(\delta)=\iint_{\Omega_{T}} d V_{\delta} \cdot \nabla \psi \mathrm{d} x \mathrm{~d} t, \quad F_{20}(\delta)=\iint_{\Omega_{T}}\left(N_{\delta}-P_{\delta}-C_{\delta}\right) \psi \mathrm{d} x \mathrm{~d} t,
$$

$$
C_{\delta}(x)=C_{K}, \quad \forall x \in K \in \mathcal{T} .
$$

Obviously, we have the strong convergence $C_{\delta} \longrightarrow C$ in $L^{\infty}(\Omega)$ as $\delta \rightarrow 0$. From the weak convergence of $\left(d V_{\delta}\right)_{\delta>0}$ to $\nabla V$ and the strong convergence of $\left(N_{\delta}, P_{\delta}\right)_{\delta>0}$ to $(N, P)$ in $L^{2}\left(\Omega_{T}\right)$, we have

$$
F_{10}(\delta)+F_{20}(\delta) \longrightarrow \iint_{\Omega_{T}} \nabla V . \nabla \psi \mathrm{d} x \mathrm{~d} t+\iint_{\Omega_{T}}(N-P-C) \psi \mathrm{d} x \mathrm{~d} t \quad \text { as } \delta \rightarrow 0 .
$$

On the other hand, multiplying the scheme (2.12) by $k \psi_{K}^{n}$ and summing for $K$ and $n$, we get

$$
F_{1}(\delta)+F_{2}(\delta)=0
$$

with

$$
F_{1}(\delta)=-\sum_{n=0}^{M_{T}} k \sum_{K \in \mathcal{T}} \sum_{L \in \mathcal{N}_{K}} d V_{K, L}^{n} \psi_{K}^{n}, \quad F_{2}(\delta)=\sum_{n=0}^{M_{T}} k \sum_{K \in \mathcal{T}} m(K)\left(N_{K}^{n}-P_{K}^{n}-C_{K}\right) \psi_{K}^{n} .
$$

Now we prove the limits $F_{j}(\delta)-F_{j 0}(\delta) \rightarrow 0$ as $\delta \rightarrow 0$ for $j=1,2$, which imply that the functions $(N, P, V)$ satisfy the Poisson equation (2.4). We start with $j=2$. A straightforward computation gives

$$
F_{2}(\delta)-F_{20}(\delta)=\sum_{n=0}^{M_{T}} \sum_{K \in \mathcal{T}} \int_{t^{n}}^{t^{n+1}} \int_{K}\left(N_{K}^{n}-P_{K}^{n}-C_{K}\right)\left(\psi_{K}^{n}-\psi(t, x)\right) \mathrm{d} x \mathrm{~d} t .
$$

Since $\left(N_{\delta}\right)_{\delta>0}$ and $\left(P_{\delta}\right)_{\delta>0}$ are bounded in $L^{\infty}\left(\Omega_{T}\right)$ and $\psi$ is smooth, it is easy to obtain

$$
\left|F_{2}(\delta)-F_{20}(\delta)\right| \leq\left[T m(\Omega)\|\psi\|_{C^{1}\left(\overline{\Omega_{T}}\right)}\left(\left\|N_{\delta}\right\|_{L^{\infty}\left(\Omega_{T}\right)}+\left\|P_{\delta}\right\|_{L^{\infty}\left(\Omega_{T}\right)}+\left\|C_{\delta}\right\|_{L^{\infty}(\Omega)}\right)\right] \delta,
$$

which yields $F_{2}(\delta)-F_{20}(\delta) \rightarrow 0$ as $\delta \rightarrow 0$. 
Next, using the definition of $d V_{K, L}^{n}$ and the symmetry of $\tau_{K, L}$, we have

$$
F_{1}(\delta)=\frac{1}{2} \sum_{n=0}^{M_{T}} k \sum_{K \in \mathcal{T}} \sum_{L \in \mathcal{N}_{K}} \tau_{K, L}\left(V_{K}^{n}-V_{L}^{n}\right)\left(\psi_{K}^{n}-\psi_{L}^{n}\right)
$$

Let us rewrite $F_{10}(\delta)$ as

$$
F_{10}(\delta)=\frac{1}{2} \sum_{n=0}^{M_{T}} \sum_{K \in \mathcal{T}} \sum_{L \in \mathcal{N}_{K}} \int_{t^{n}}^{t^{n+1}} \int_{T_{K, L}} d V_{\delta} . \nabla \psi \mathrm{d} x \mathrm{~d} t
$$

Therefore, by the definition of $\tau_{K, L}$ and $d V_{\delta}$,

$$
F_{1}(\delta)-F_{10}(\delta)=\frac{1}{2} \sum_{n=0}^{M_{T}} \sum_{K \in \mathcal{T}} \sum_{L \in \mathcal{N}_{K}} m\left(\sigma_{K L}\right)\left(V_{K}^{n}-V_{L}^{n}\right) \int_{t^{n}}^{t^{n+1}}\left(\frac{\psi_{K}^{n}-\psi_{L}^{n}}{d\left(x_{K}, x_{L}\right)}-\frac{1}{m\left(T_{K, L}\right)} \int_{T_{K, L}} \nabla \psi \cdot \nu_{L, K} \mathrm{~d} x\right) \mathrm{d} t .
$$

Since the straight line $\overline{x_{K} x_{L}}$ is orthogonal to $\sigma_{K L}$, we have $x_{K}-x_{L}=d\left(x_{K}, x_{L}\right) \nu_{L, K}$. It follows from the regularity of $\psi$ that

$$
\begin{aligned}
\frac{\psi_{K}^{n}-\psi_{L}^{n}}{d\left(x_{K}, x_{L}\right)} & =\nabla \psi\left(t^{n}, x_{L}\right) \cdot \nu_{L, K}+O(h) \\
& =\nabla \psi(t, x) \cdot \nu_{L, K}+O(\delta), \quad \forall(t, x) \in\left(t^{n}, t^{n+1}\right) \times T_{K, L}
\end{aligned}
$$

By taking the mean value over $T_{K, L}$, there is a constant $D_{7}>0$ depending only on $\psi$ such that

$$
\left|\int_{t^{n}}^{t^{n+1}}\left(\frac{\psi_{K}^{n}-\psi_{L}^{n}}{d\left(x_{K}, x_{L}\right)}-\frac{1}{m\left(T_{K, L}\right)} \int_{T_{K, L}} \nabla \psi \cdot \nu_{L, K} \mathrm{~d} x\right) \mathrm{d} t\right| \leq D_{7} k \delta
$$

On the other hand, using (4.16),

$$
\begin{aligned}
m\left(\sigma_{K L}\right)\left|V_{K}^{n}-V_{L}^{n}\right| & =\sqrt{\tau_{K, L}}\left|V_{K}^{n}-V_{L}^{n}\right| \sqrt{d\left(x_{K}, x_{L}\right) m\left(\sigma_{K L}\right)} \\
& \leq \sqrt{\tau_{K, L}}\left|V_{K}^{n}-V_{L}^{n}\right| \sqrt{D_{6} m\left(T_{K, L}\right)} .
\end{aligned}
$$

Hence, by Cauchy-Schwarz inequality, the discrete $L^{\infty}\left(0, T ; H^{1}(\Omega)\right)$ estimate (4.1) and (4.17), we obtain

$$
\left|F_{1}(\delta)-F_{10}(\delta)\right| \leq D_{8} \delta,
$$

where $D_{8}>0$ is a constant depending only on $D_{1}, D_{6}$ and $D_{7}$. This shows that $F_{1}(\delta)-F_{10}(\delta) \rightarrow 0$ as $\delta \rightarrow 0$.

Now it remains to show that $V-\bar{V} \in L^{\infty}\left(0, T ; H_{0}^{1}(\Omega)\right)$. Let us introduce $W_{\delta}=V_{\delta}-\bar{V}_{\delta}$ in $\Omega_{T}$, where $\bar{V}_{\delta}$ is the discrete function of $\bar{V}$ defined in the proof of Lemma 4.1. Since $W_{\delta}=0$ on $\Gamma$, we extend $W_{\delta}$ to the whole space $(0, T) \times \mathbb{R}^{d}$ such that for almost all $t \in(0, T), W_{\delta}(., t)=0$ in $\mathbb{R}^{d} \backslash \Omega$. This extension is still denoted by $W_{\delta}$. From the definition of $\bar{V}_{\delta}$, it is easy to see that as $\delta \rightarrow 0$,

$$
\bar{V}_{\delta} \longrightarrow \bar{V} \text { in } L^{2}\left(\Omega_{T}\right) \text { strongly. }
$$

On the other hand, from the discrete $L^{\infty}\left(0, T ; H^{1}(\Omega)\right)$ estimate (4.1) and Lemma 4.3 of [10], we obtain for almost all $t \in(0, T)$ and any $\eta \in \mathbb{R}^{d}$ that,

$$
\left\|W_{\delta}(t, .+\eta)-W_{\delta}(t, .)\right\|_{L^{2}\left(\mathbb{R}^{d}\right)}^{2} \leq D_{9}|\eta|(|\eta|+h+1) .
$$


This shows by the criterion of strong compactness [3] that for almost all $t \in(0, T)$, a subsequence of $\left(W_{\delta}(t, .)\right)_{\delta>0}$ converges to $W(t,$.$) in L^{2}\left(\mathbb{R}^{d}\right)$ strongly as $\delta \rightarrow 0$. It is necessary that the restriction of $W(., t)$ to $\Omega$ belongs to $H_{0}^{1}(\Omega)$. From the uniqueness of the limit, we get that $W=V-\bar{V} \in L^{\infty}\left(0, T ; H_{0}^{1}(\Omega)\right)$. This completes the proof.

Theorem 5.2. Assume (H1)-(H4) hold. Then the functions $(N, P, V)$ defined in Lemmas 4.3-4.4 satisfy the continuity equations in the sense of (2.2)-(2.3). Moreover, $N-\bar{N}, P-\bar{P} \in L^{2}\left(0, T ; H_{0}^{1}(\Omega)\right)$.

Proof. The proof of $N-\bar{N}, P-\bar{P} \in L^{2}\left(0, T ; H_{0}^{1}(\Omega)\right)$ is similar to that of $V-\bar{V} \in L^{\infty}\left(0, T ; H_{0}^{1}(\Omega)\right)$. The proof of (2.2) and (2.3) is almost the same. Therefore it suffices to show (2.2). As above, the subscript $N$ in $r_{N}$ will be omitted in the proof.

Let $\phi \in \mathcal{D}([0, T) \times \Omega)$ be a test function and $\delta>0$ be small enough such that $\operatorname{supp} \phi \in\left[0,\left(M_{T}-1\right) k\right) \times\{x \in$ $\Omega ; d(x, \Gamma)>\delta\}$. We define

$$
\begin{aligned}
G_{10}(\delta) & =-\iint_{\Omega_{T}} N_{\delta} \partial_{t} \phi \mathrm{d} x \mathrm{~d} t-\int_{\Omega} N_{\delta}(0, x) \phi(0, x) \mathrm{d} x, \\
G_{20}(\delta) & =\iint_{\Omega_{T}} d r\left(N_{\delta}\right) \cdot \nabla \phi \mathrm{d} x \mathrm{~d} t \\
G_{30}(\delta) & =-\iint_{\Omega_{T}} N_{\delta} d V_{\delta} \cdot \nabla \phi \mathrm{d} x \mathrm{~d} t
\end{aligned}
$$

and

$$
\varepsilon(\delta)=-\left[G_{10}(\delta)+G_{20}(\delta)+G_{30}(\delta)\right] .
$$

Let $\phi_{K}^{n}=\phi\left(t^{n}, x_{K}\right)$ for all $K \in \mathcal{T}$ and $n=0,1, \ldots, M_{T}$. Multiplying the scheme (2.10) by $k \phi_{K}^{n}$ and summing for $K$ and $n$, we obtain

$$
G_{1}(\delta)+G_{2}(\delta)+G_{3}(\delta)=0
$$

where

$$
\begin{aligned}
G_{1}(\delta) & =\sum_{n=0}^{M_{T}} \sum_{K \in \mathcal{T}} m(K)\left(N_{K}^{n+1}-N_{K}^{n}\right) \phi_{K}^{n}, \\
G_{2}(\delta) & =\sum_{n=0}^{M_{T}} k \sum_{K \in \mathcal{T}} \sum_{L \in \mathcal{N}_{K}} \tau_{K, L}\left[r\left(N_{K}^{n+1}\right)-r\left(N_{L}^{n+1}\right)\right] \phi_{K}^{n}, \\
G_{3}(\delta) & =\sum_{n=0}^{M_{T}} k \sum_{K \in \mathcal{T}} \sum_{L \in \mathcal{N}_{K}}\left[\left(d V_{K, L}^{n}\right)^{+} N_{K}^{n+1}+\left(d V_{K, L}^{n}\right)^{-} N_{L}^{n+1}\right] \phi_{K}^{n} .
\end{aligned}
$$

From the weak convergence of the sequences $\left(d V_{\delta}\right)_{\delta>0}$ to $\nabla V,\left(d r\left(N_{\delta}\right)\right)_{\delta>0}$ to $\nabla r(N)$ and the strong convergence of the sequence $\left(N_{\delta}\right)_{\delta>0}$ to $N$ in $L^{2}\left(\Omega_{T}\right)$, it is easy to see that,

$$
\varepsilon(\delta) \longrightarrow \iint_{\Omega_{T}}\left(N \partial_{t} \phi-\nabla r(N) . \nabla \phi+N \nabla V . \nabla \phi\right) \mathrm{d} x \mathrm{~d} t+\int_{\Omega} N^{0}(x) \phi(0, x) \mathrm{d} x \quad \text { as } \delta \rightarrow 0 .
$$

Therefore, it remains to show that $\varepsilon(\delta) \rightarrow 0$ as $\delta \rightarrow 0$, which will be achieved from the limits: $G_{j}(\delta)-G_{j 0}(\delta) \rightarrow 0$ as $\delta \rightarrow 0, j=1,2,3$. In view of the expression of $G_{2}(\delta)$ and $G_{20}(\delta)$, it is easy to see that the proof of $G_{2}(\delta)-G_{20}(\delta) \rightarrow 0$ is similar to that of $F_{1}(\delta)-F_{10}(\delta) \rightarrow 0$ in Theorem 5.1. Hence we only show $G_{1}(\delta)-G_{10}(\delta) \rightarrow$ 0 and $G_{3}(\delta)-G_{30}(\delta) \rightarrow 0$ as $\delta \rightarrow 0$. 
For the first limit, we have

$$
\begin{aligned}
G_{1}(\delta) & =\sum_{n=0}^{M_{T}} \sum_{K \in \mathcal{T}} m(K) N_{K}^{n+1}\left(\phi_{K}^{n}-\phi_{K}^{n+1}\right)-\sum_{K \in \mathcal{T}} m(K) N_{K}^{0} \phi_{K}^{0} \\
& =-\sum_{n=0}^{M_{T}} \sum_{K \in \mathcal{T}} \int_{t^{n}}^{t^{n+1}} \int_{K} N_{K}^{n+1} \partial_{t} \phi\left(t, x_{K}\right) \mathrm{d} x \mathrm{~d} t-\sum_{K \in \mathcal{T}} \int_{K} N_{K}^{0} \phi\left(0, x_{K}\right) \mathrm{d} x, \\
G_{10}(\delta) & =-\sum_{n=0}^{M_{T}} \sum_{K \in \mathcal{T}} \int_{t^{n}}^{t^{n+1}} \int_{K} N_{K}^{n+1} \partial_{t} \phi(t, x) \mathrm{d} x \mathrm{~d} t-\sum_{K \in \mathcal{T}} \int_{K} N_{K}^{0} \phi(0, x) \mathrm{d} x .
\end{aligned}
$$

Hence, it follows from the regularity of $\phi$ that

$$
\left|G_{1}(\delta)-G_{10}(\delta)\right| \leq\left[(T+1) m(\Omega)\left\|N_{\delta}\right\|_{L^{\infty}\left(\Omega_{T}\right)}\|\phi\|_{C^{2}\left(\overline{\Omega_{T}}\right)}\right] h \longrightarrow 0 \quad \text { as } \delta \rightarrow 0
$$

For the second limit, using the relation

$$
\left(d V_{K, L}^{n}\right)^{+} N_{K}^{n+1}+\left(d V_{K, L}^{n}\right)^{-} N_{L}^{n+1}=\frac{1}{2}\left|d V_{K, L}^{n}\right|\left(N_{K}^{n+1}-N_{L}^{n+1}\right)+\frac{1}{2} d V_{K, L}^{n}\left(N_{K}^{n+1}+N_{L}^{n+1}\right),
$$

we may write $G_{3}(\delta)=G_{31}(\delta)+G_{32}(\delta)$, with

$$
\begin{aligned}
G_{31}(\delta) & =\frac{1}{2} \sum_{n=0}^{M_{T}} k \sum_{K \in \mathcal{T}} \sum_{L \in \mathcal{N}_{K}}\left|d V_{K, L}^{n}\right|\left(N_{K}^{n+1}-N_{L}^{n+1}\right) \phi_{K}^{n} \\
& =\frac{1}{4} \sum_{n=0}^{M_{T}} k \sum_{K \in \mathcal{T}} \sum_{L \in \mathcal{N}_{K}}\left|d V_{K, L}^{n}\right|\left(N_{K}^{n+1}-N_{L}^{n+1}\right)\left(\phi_{K}^{n}-\phi_{L}^{n}\right), \\
G_{32}(\delta) & =\frac{1}{2} \sum_{n=0}^{M_{T}} k \sum_{K \in \mathcal{T}} \sum_{L \in \mathcal{N}_{K}} d V_{K, L}^{n}\left(N_{K}^{n+1}+N_{L}^{n+1}\right) \phi_{K}^{n} \\
& =\frac{1}{2} \sum_{n=0}^{M_{T}} k \sum_{K \in \mathcal{T}} \sum_{L \in \mathcal{N}_{K}} N_{K}^{n+1} d V_{K, L}^{n}\left(\phi_{K}^{n}-\phi_{L}^{n}\right) .
\end{aligned}
$$

From the definition of $N_{\delta}$, we have also

$$
\begin{aligned}
G_{30}(\delta) & =-\frac{1}{2} \sum_{n=0}^{M_{T}} \sum_{K \in \mathcal{T}} \sum_{L \in \mathcal{N}_{K}} \int_{t^{n}}^{t^{n+1}} \int_{T_{K, L}} N_{\delta} d V_{\delta} \cdot \nabla \phi \mathrm{d} x \mathrm{~d} t \\
& =G_{310}(\delta)+G_{320}(\delta)
\end{aligned}
$$

with

$$
\begin{aligned}
& G_{310}(\delta)=-\frac{1}{2} \sum_{n=0}^{M_{T}} \sum_{K \in \mathcal{T}} \sum_{L \in \mathcal{N}_{K}} \int_{t^{n}}^{t^{n+1}} \int_{S_{L, K}}\left(N_{L}^{n+1}-N_{K}^{n+1}\right) d V_{\delta} \cdot \nabla \phi \mathrm{d} x \mathrm{~d} t \\
& G_{320}(\delta)=-\frac{1}{2} \sum_{n=0}^{M_{T}} \sum_{K \in \mathcal{T}} \sum_{L \in \mathcal{N}_{K}} \int_{t^{n}}^{t^{n+1}} \int_{T_{K, L}} N_{K}^{n+1} d V_{\delta} \cdot \nabla \phi \mathrm{d} x \mathrm{~d} t
\end{aligned}
$$


where $S_{L, K}=L \cap T_{K, L}$. Therefore, the convergence result follows if we prove that $G_{31}(\delta) \rightarrow 0, G_{310}(\delta) \rightarrow 0$ and $G_{32}(\delta)-G_{320}(\delta) \rightarrow 0$ as $\delta \rightarrow 0$.

First of all, by Cauchy-Schwarz inequality and using (4.1)-(4.2), we obtain

$$
\left|G_{31}(\delta)\right| \leq \frac{h}{2} \sqrt{T D_{1}}\|\phi\|_{C^{1}\left(\overline{\Omega_{T}}\right)}\left\|N_{\delta}\right\|_{1, \Omega_{T}} \longrightarrow 0 \quad \text { as } \delta \rightarrow 0 .
$$

Next, noting that $d V_{\delta}=\frac{d\left(x_{K}, x_{L}\right)}{m\left(T_{K, L}\right)} d V_{K, L} \cdot \nu_{K, L}$ in $T_{K, L}$ and $S_{L, K} \subset T_{K, L}$, we obtain again from Cauchy-Schwarz inequality,

$$
\begin{aligned}
\left|G_{310}(\delta)\right| & \leq \frac{1}{2} \sum_{n=0}^{M_{T}} k \sum_{K \in \mathcal{T}} \sum_{L \in \mathcal{N}_{K}} d\left(x_{K}, x_{L}\right)\left|d V_{K, L}\right|\left|N_{K}^{n+1}-N_{L}^{n+1}\right|\|\phi\|_{C^{1}\left(\overline{\Omega_{T}}\right)} \\
& \leq h \sqrt{T D_{1}}\|\phi\|_{C^{1}\left(\overline{\Omega_{T}}\right)}\left\|N_{\delta}\right\|_{1, \Omega_{T}} \longrightarrow 0 \quad \text { as } \delta \rightarrow 0 .
\end{aligned}
$$

Finally,

$$
\begin{aligned}
& G_{32}(\delta)-G_{320}(\delta)= \\
& \quad \frac{1}{2} \sum_{n=0}^{M_{T}} \sum_{K \in \mathcal{T}} \sum_{L \in \mathcal{N}_{K}} m\left(\sigma_{K L}\right) N_{K}^{n+1}\left(V_{K}^{n}-V_{L}^{n}\right) \int_{t^{n}}^{t^{n+1}}\left(\frac{\phi_{L}^{n}-\phi_{K}^{n}}{d\left(x_{K}, x_{L}\right)}-\frac{1}{m\left(T_{K, L}\right)} \int_{T_{K, L}} \nabla \phi . \nu_{K, L} \mathrm{~d} x\right) \mathrm{d} t .
\end{aligned}
$$

Using the $L^{\infty}\left(\Omega_{T}\right)$ bound for $N_{\delta}$, we obtain $G_{32}(\delta)-G_{320}(\delta) \rightarrow 0$ as $\delta \rightarrow 0$ similarly to that of $F_{1}(\delta)-F_{10}(\delta) \rightarrow 0$. This ends the proof of Theorem 5.2.

\section{Numerical example}

In this last section, we give a numerical result in two space dimensions for the simulation of a reverse biased diode. The data are picked in the paper [18], so that the results by the finite volume scheme can be compared to those obtained by the mixed exponential fitting method.

In our example, the particle densities and the electrostatic potential are given at the ohmic contact $\Gamma_{\mathrm{D}}$ (Dirichlet boundary) and the remaining part of the boundary $\Gamma_{N}=\Gamma \backslash \Gamma_{\mathrm{D}}$ is insulating (Neumann boundary). The device is made of a $P$-region where the preconcentration of electrons dominates $(C<0)$ and a $N$-region where the preconcentration of holes dominates $(C>0)$. The dimensionless doping profile is taken as piecewise constants:

$$
C=-1 \text { in the } P \text {-region and } C=1 \text { in the } N \text {-region. }
$$

The device is assumed to be a square of size $10^{-3} \mathrm{~cm}$. The solutions are computed with a $42 \times 42$ uniform Cartesian grid for a large $T$ in order to obtain the steady-state solution (which does not depend on the initial conditions). Figure 1 illustrates the carrier densities and electrostatic potential with the level curves for the carrier densities. It is easy to see that the vacuum sets occur for carrier densities. Therefore, no current flows through the diode. Figure 1 shows that our scheme is numerical stable and well-adapted in the presence of the vacuum sets for the carrier densities. We refer to [8] for more detail and more numerical results.

Acknowledgements. This work was carried out during the visit of J.G. Liu in Laboratoire de Mathématiques Appliquées, Université Blaise Pascal in January 2002. He is grateful to the members of the Laboratoire for their hospitality. The research of J.G. Liu was partially supported by NSF Grant DMS-0107218. C. Chainais-Hillairet and Y.J. Peng acknowledge partial support from the European IHP project "Hyperbolic and Kinetic Equations", contract number HPRN-CT-200200282 . 


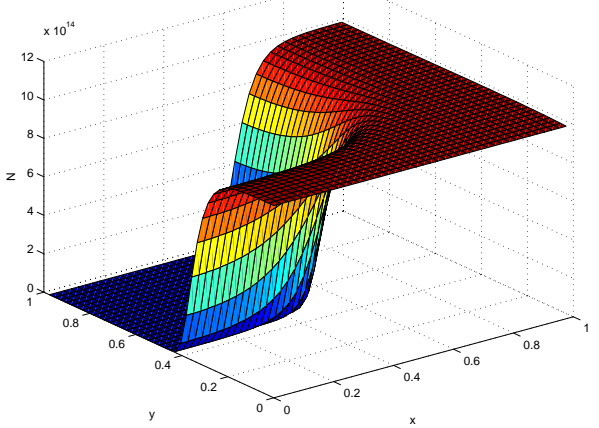

(a) Electron density

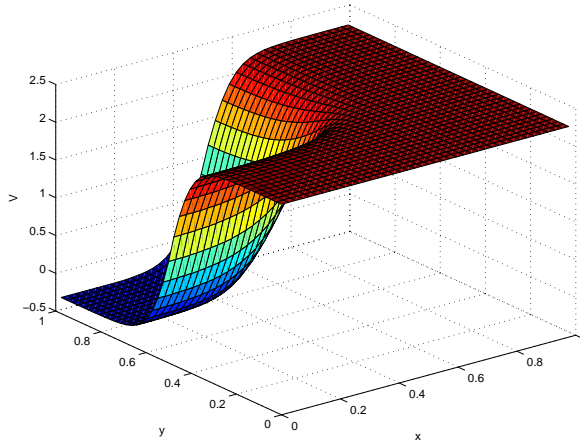

(c) Electrostatic potential

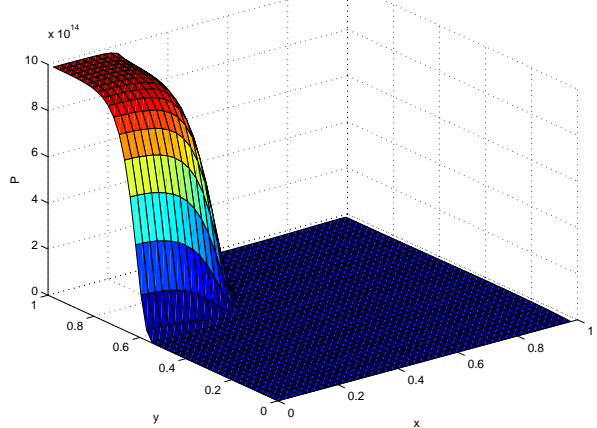

(b) Hole density

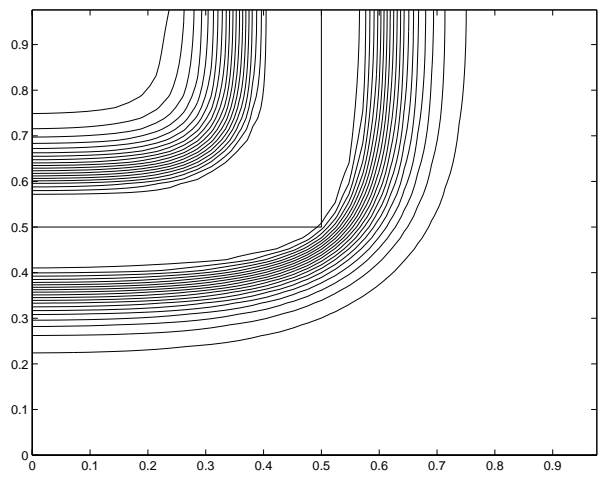

(d) Level curves of $N$ and $P$

FiguRE 1. Solutions computed at $T=4$.

\section{REFERENCES}

[1] F. Arimburgo, C. Baiocchi and L.D. Marini, Numerical approximation of the 1-D nonlinear drift-diffusion model in semiconductors, in Nonlinear kinetic theory and mathematical aspects of hyperbolic systems, Rapallo, (1992) 1-10. World Sci. Publishing, River Edge, NJ (1992).

[2] H. Beirão da Veiga, On the semiconductor drift diffusion equations. Differential Integral Equations 9 (1996) 729-744.

[3] H. Brezis, Analyse Fonctionnelle - Théorie et Applications. Masson, Paris (1983).

[4] F. Brezzi, L.D. Marini and P. Pietra, Méthodes d'éléments finis mixtes et schéma de Scharfetter-Gummel. C. R. Acad. Sci. Paris Sér. I Math. 305 (1987) 599-604.

[5] F. Brezzi, L.D. Marini and P. Pietra, Two-dimensional exponential fitting and applications to drift-diffusion models. SIAM J. Numer. Anal. 26 (1989) 1342-1355.

[6] C. Chainais-Hillairet and Y.J. Peng, Convergence of a finite volume scheme for the drift-diffusion equations in 1-D. IMA J. Numer. Anal. 23 (2003) 81-108.

[7] C. Chainais-Hillairet and Y.J. Peng, A finite volume scheme to the drift-diffusion equations for semiconductors, in Proc. of The Third International Symposium on Finite Volumes for Complex Applications, R. Herbin and D. Kröner Eds., Hermes, Porquerolles, France (2002) 163-170.

[8] C. Chainais-Hillairet and Y.J. Peng, Finite volume approximation for degenerate drift-diffusion system in several space dimensions. Math. Models Methods. Appl. Sci. (submitted).

[9] P.G. Ciarlet, The Finite Element Method for Elliptic Problems. North-Holland, Amsterdam (1978).

[10] R. Eymard, T. Gallouët and R. Herbin, Finite Volume Methods. North-Holland, Amsterdam, Handb. Numer. Anal. VII (2000) 713-1020.

[11] R. Eymard, T. Gallouët, R. Herbin and A. Michel, Convergence of a finite volume scheme for nonlinear degenerate parabolic equations. Numer. Math. 92 (2002) 41-82. 
[12] W. Fang and K. Ito, Global solutions of the time-dependent drift-diffusion semiconductor equations. J. Differential Equations 123 (1995) 523-566.

[13] H. Gajewski, On the uniqueness of solutions to the drift-diffusion model of semiconductor devices. Math. Models Methods Appl. Sci. 4 (1994) 121-133.

[14] A. Jüngel, Numerical approximation of a drift-diffusion model for semiconductors with nonlinear diffusion. ZAMM Z. Angew. Math. Mech. 75 (1995) 783-799.

[15] A. Jüngel, A nonlinear drift-diffusion system with electric convection arising in semiconductor and electrophoretic modeling. Math. Nachr. 185 (1997) 85-110.

[16] A. Jüngel and Y.J. Peng, A hierarchy of hydrodynamic models for plasmas: zero-relaxation-time limits. Comm. Partial Differential Equations 24 (1999) 1007-1033.

[17] A. Jüngel and Y.J. Peng, Zero-relaxation-time limits in the hydrodynamic equations for plasmas revisited. Z. Angew. Math. Phys. 51 (2000) 385-396.

[18] A. Jüngel and P. Pietra, A discretization scheme for a quasi-hydrodynamic semiconductor model. Math. Models Methods Appl. Sci. 7 (1997) 935-955.

[19] P.A. Markowich, C.A. Ringhofer and C. Schmeiser, Semiconductor Equations. Springer-Verlag, Vienna (1990).

To access this journal online: www.edpsciences.org 